Honam Mathematical J. 35 (2013), No. 4, pp. 765-773

http://dx.doi.org/10.5831/HMJ.2013.35.4.765

\title{
NORMAL NUMBERS MOD 2 ON THE LOGISTIC MAP
}

\author{
YounG-Ho AHN
}

\begin{abstract}
We show that for the logistic map, almost every $x$ is a normal number mod 2 with respect to all intervals except for $[a, b]=\left[\frac{1}{4}, 1\right]$ or $[a, b]=\left[\frac{1}{2}-\frac{\sqrt{3}}{4}, \frac{1}{2}+\frac{\sqrt{3}}{4}\right]$.
\end{abstract}

\section{Introduction}

Let $(X, \mu)$ be a probability measure space. A measurable transformation $T: X \rightarrow X$ is said to be measure preserving if $\mu\left(T^{-1} E\right)=\mu(E)$ for every measurable subset $E$. A measure preserving transformation $T$ on $X$ is called ergodic if $f(T x)=f(x)$ holds only for constant functions.

Let $\mathbf{1}_{E}$ be the characteristic function of a set $E$ and consider the behavior of the sequence $\sum_{k=0}^{n-1} \mathbf{1}_{E}\left(T^{k} x\right)$ which equals the number of times that the points $T^{k} x$ visit $E$. The Birkhoff Ergodic Theorem applied to the ergodic transformation $T: x \mapsto\{2 x\}$ on $[0,1)$, where $\{t\}$ is the fractional part of $t$, gives the classical Borel's Theorem on normal numbers:

$$
\lim _{n \rightarrow \infty} \frac{1}{n} \sum_{k=0}^{n-1} \mathbf{1}_{\left[\frac{1}{2}, 1\right)}\left(T^{k} x\right)=\frac{1}{2} .
$$

This implies that a.e. $x$ is normal, i.e., the relative frequency of the digit 1 in the binary expansion of $x$ is $\frac{1}{2}$, see [7].

Recall that the logistic transformation, $T$ on $[0,1]$ defined by $x \rightarrow$ $4 x(1-x)$. In this paper, we are interested in the uniform distribution of the sequence $d_{n} \in\{0,1\}$ defined by

$$
d_{n}(x) \equiv \sum_{k=0}^{n-1} \mathbf{1}_{E}\left(T^{k} x\right) \quad(\bmod 2),
$$

Received October 23, 2013. Accepted November 11, 2013. 2010 Mathematics Subject Classification. 28D05, 47A35.

Key words and phrases. normal number mod 2, logistic map, tent map, ergodic. 
for the logistic map on the interval where $E$ is a finite union of intervals. We say that $x$ is a normal number mod 2 with respect to $E$ and the transformation $T$ if

$$
\lim _{N \rightarrow \infty} \frac{1}{N} \sum_{n=1}^{N} d_{n}(x)=\frac{1}{2} .
$$

Contrary to our intuition, the limit might not exist and even when it exists it may not be equal to $\frac{1}{2}$. This type of problem was first studied by Veech[6]. He considered the case when the transformations are given by irrational rotations on the unit circle, and obtained results which showed that the length of the interval $E$ and the rotational angle $\theta$ are closely related. For example, he proved that when the irrational number $\theta$ has bounded partial quotients in its continued fraction expansion, the sequence $d_{n}$ is evenly distributed if the length of the interval is not an integral multiple of $\theta$ modulo 1 . For a related result, see [4].

In $[1,2]$, the authors consider the case when transformations defined by $x \mapsto L x(\bmod 1)$ with $L \in \mathbb{R}$ on $X=[0,1)$ and show that the sequence $\left\{d_{n}\right\}$ is evenly distributed if $\exp \left(\pi i \mathbf{1}_{E}(x)\right)$ has finite $L$-adic discontinuity points $\frac{1}{L} \leq t_{1}<\cdots<t_{n} \leq 1$. Recently, Choe, Hamachi and Nakada [4] show that $\left\{d_{n}\right\}$ is evenly distributed for more general sets and that $\mathbb{Z}_{2}$-extension induced by $\phi(x)=\exp \left(\pi i \mathbf{1}_{B}(x)\right)$ where $\mathbf{1}_{B}$ is a characteristic function of $B$, is ergodic.

Recall that the transformations which are considered in $[1,2,6]$ are Lebesgue measure preserving or its invariant density function is bounded. Contrast to those, the logistic map does not preserve Lebesgue measure and also its invariant density function is not bounded.

\section{Properties of the logistic map}

Recall that the logistic map $S$ on $[0,1]$ is defined by

$$
S x=4 x(1-x)
$$

and its invariant probability density function of $S$ is given by

$$
\rho(x)=\frac{1}{\pi \sqrt{x(1-x)}},
$$

i.e., the measure $\nu$ defined by

$$
\nu(E)=\int_{E} \rho(x) d x
$$


is an $S$-invariant probability measure. To show that it is enough to consider the class of the sets of the form $E=(b, 1)$ with $0<b<1$, because that class generates the Borel $\sigma$-algebra on $[0,1)$.

Since

$$
\nu(E)=\int_{b}^{1} \frac{1}{\pi \sqrt{x(1-x)}} d x=\frac{1}{2}-\frac{\arcsin (2 b-1)}{\pi}
$$

and

$$
\nu\left(S^{-1} E\right)=\int_{S^{-1} E} \rho(x) d x=\int_{\frac{1-\sqrt{1-b}}{2}}^{\frac{1+\sqrt{1-b}}{2}} \frac{1}{\pi \sqrt{x(1-x)}} d x=\frac{2 \arcsin (\sqrt{1-b})}{\pi},
$$

$\nu\left(S^{-1} E\right)=\nu(E)$. Hence $S$ has the absolutely continuous invariant probability measure but its density function is not bounded.

Let $T_{1}:\left(X_{1}, \mu_{1}\right) \rightarrow\left(X_{1}, \mu_{1}\right)$ and $T_{2}:\left(X_{2}, \mu_{2}\right) \rightarrow\left(X_{2}, \mu_{2}\right)$ be measure preserving. Two measure preserving transformations are said to be measure theoretically isomorphic if there exists an isomorphism $\psi:\left(X_{1}, \mu_{1}\right) \rightarrow\left(X_{2}, \mu_{2}\right)$ such that $\psi \circ T_{1}=T_{2} \circ \psi$, in other words, the following diagram commutes:

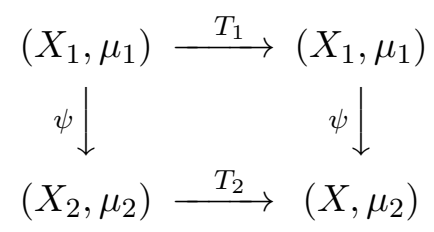

Recall that the tent map $T$ on $[0,1]$ is defined by

$$
T x=\left\{\begin{array}{ll}
2 x, & \text { if } 0 \leq x \leq \frac{1}{2} \\
2-2 x, & \text { otherwise }
\end{array} .\right.
$$

The following lemma shows that the logistic map and the tent map are measure theoretically isomorphic.

Lemma 1. The tent map $T$ on $([0,1], \mu)$ and the logistic map $S$ on $([0,1], \nu)$ are measure theoretically isomorphic where $\mu$ is Lebesgue measure on $[0,1]$. Furthermore there exists an isomorphism whis is also a topological homeomorphism.

Proof. Note that the tent transformaion $T$ preserves Lebesuge measure. 
Define $\psi(x)=\sin ^{2}\left(\frac{\pi x}{2}\right)=\frac{1-\cos (\pi x)}{2}$ on $[0,1]$. Then $\psi$ is an homeomorphism on $[0,1]$ and also preserves the order on $[0,1]$. Since

$$
a=\mu\left(\psi^{-1}([0, \psi(a)])=\int_{0}^{\sin ^{2}(\pi a / 2)} \rho(x) d x=\nu([0, \psi(a)]),\right.
$$

$\psi:([0,1], \mu) \rightarrow([0,1], \nu)$ is measure preserving. Furthermore

$$
S \psi(x)=4 \sin ^{2}\left(\frac{\pi x}{2}\right)\left(1-\sin ^{2}\left(\frac{\pi x}{2}\right)\right)=\sin ^{2}(\pi x)=\psi(T x) .
$$

Thus the following diagram commutes and hence $S$ and $T$ are measure theoretically isomorphic.

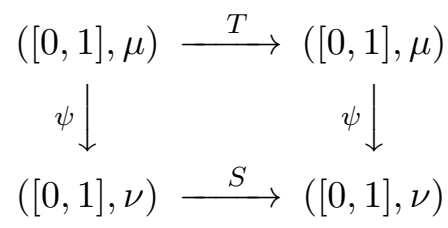

\section{Normal numbers mod 2}

We investigate the problem from the viewpoint of spectral theory. Let $(X, \mu)$ be a probability space and $T$ an ergodic measure preserving transformation on $X$, which is not necessarily invertible. To investigate the sequence $\left\{d_{n}(x)\right\}$, we consider the behavior of the sequence $\exp \left(\pi i d_{n}(x)\right)$ and check whether this sequence is uniformly distributed on a compact group $G=\{-1,1\}$. Let $\phi(x)$ be a $G$-valued function defined by $\phi(x)=\exp \left(\pi i \mathbf{1}_{E}(x)\right)$. Consider the skew product transformation $T_{\phi}$ on $X \times G$ defined by

$$
T_{\phi}(x, g)=(T x, \phi(x) g) .
$$

Then the problem is equivalent to checking whether $T_{\phi}$ is ergodic or not.

To check whether $\lim _{N \rightarrow \infty} \frac{1}{N} \sum_{n=1}^{N} \exp \left(\pi i d_{n}(x)\right)=0$ or not, consider a skew product transformation $T_{\phi}$ on $[0,1) \times\{-1,1\}$ defined by $T_{\phi}(x, z)=(T x, \phi(x) \cdot z)$. Then

$$
\lim _{N \rightarrow \infty} \frac{1}{N} \sum_{1}^{N} \exp \left(\pi i d_{n}(x)\right) \cdot z=\lim _{N \rightarrow \infty} \frac{1}{N} \sum_{1}^{N} U_{T_{\phi}} f(x, z)
$$

where $U_{T_{\phi}}$ is an isometry on $L^{2}(X \times\{-1,1\})$ induced by $T_{\phi}$ and $f(x, z)=$ $z$. Hence if $T_{\phi}$ is ergodic, then $\lim _{N \rightarrow \infty} \frac{1}{N} \sum_{1}^{N} \exp \left(\pi i d_{n}(x)\right)=0$ by an application of the Birkhoff Ergodic theorem to $f(x, z)=z$. If $T_{\phi}$ is not 
ergodic, then there exists $q(x)$ such that $q(x)=\exp \left(\pi i \mathbf{1}_{F}(x)\right)$ for some measurable set $F$ and $\exp \left(\pi i \mathbf{1}_{E}(x)\right)=q(x) q(T x)$. Furthermore,

$$
\lim _{N \rightarrow \infty} \frac{1}{N} \sum_{1}^{N} \exp \left(\pi i d_{n}(x)\right)=q(x) \int_{[0,1)} q(t) d \mu(t) .
$$

For related results, see [3].

Lemma 2. For the logistic map, if a $G$-valued function $\phi(x)$ is a step function with finite discontinuity points and $\phi(x) q(S x)=q(x)$, then there exists $G$-valued function $\hat{q}(x)$ which is a step function with finite discontinuity points and $\phi(x) \hat{q}(S x)=\hat{q}(x)$. Hence we may assume that $q(x)$ is a $G$-valued step function with finite discontinuity points.

Proof. Recall that the tent map $T$ on $([0,1], \mu)$ and the logistic map $S$ on $([0,1], \nu)$ are measure theoretically isomorphic via the topological homeomorphism $\psi(x)=\sin ^{2}\left(\frac{\pi x}{2}\right)$ by Lemma 1 .

Let $f(x)=\phi(\psi(x))$ and $h(x)=q(\psi x)$. Then

$$
f(x) h(T x)=h(x)
$$

and since $\psi(x)$ is also a topological homeomorphism, $f(x)$ is also a step function with finite discontinuity points. Hence it is enough to show that $h(x)$ is also a step function with finite discontinuity points.

Since $|f(x)|=1$ for a.e. $x \in[0,1)$ and $T$ is ergodic transformation, we may assume that $|h(x)|=1$ for a.e. $x \in[0,1)$ and furthermore we also may assume that $h(x)$ is also a $G$-valued function. Let $\mathcal{P}$ be a partition defined by $\mathcal{P}=\left\{\left[0, \frac{1}{2}\right),\left[\frac{1}{2}, 1\right)\right\}$, and $\mathcal{P}_{N}=\bigvee_{k=0}^{N-1} T^{-k} \mathcal{P}$. Let $D=$ $\{z \mid f(x)$ is discontinuous at $x=z\}, m$ be the cardinality of discontinuity $D$ and $D_{\epsilon}$ be the $\epsilon$-neighborhood of $D$, i.e., $D_{\epsilon}=\{(z-\epsilon, z+\epsilon) \mid z \in D\}$ . Then there exists $\epsilon_{0}$ such that for all $0<\epsilon<\epsilon_{0}, \mu\left(D_{\epsilon}\right)=2 m \epsilon$. Now choose an integer $N$ such that $\frac{1}{2}^{N}<\epsilon_{0}$ and $m \cdot \frac{1}{2}^{N-1}<\frac{1}{2}$.

If $I \in \mathcal{P}_{N}$ and if $I \cap D \neq \phi$, then $I \subset D_{\epsilon}$ for $\epsilon=\frac{1}{2}^{N}$. Hence the totality of $I \in \mathcal{P}_{N}$ with $I \cap D \neq \phi$ measure at most $2 m \cdot \frac{1}{2}^{N}$. By the similar argument, the totality of $I \in \mathcal{P}_{N+j}, j \geq 0$ such that $I \cap D \neq \phi$ measure at most $2 m \cdot \frac{1}{2}^{N+j}$.

Fix $L>0$ and consider the collection of $I \in \mathcal{P}_{N+L}$ having the property that $T^{j} I \cap D \neq \phi$ for some $0 \leq j \leq L-1$. Since $T^{j} \in \mathcal{P}_{N+L-j}$ for these $j$, and $T$ is measure preserving, these intervals have a total measure at most

$$
\sum_{j=1}^{L-1} 2 m \cdot \frac{1}{2}^{N+L-j} \leq m \cdot \frac{1}{2}^{N-1} \leq \frac{1}{2} .
$$


Let $Q(N, L)$ be the sub collection of $\mathcal{P}_{N+L}$ such that $T^{j} I \cap Y=\phi$ for all $0 \leq j \leq L-1$. Then for each $I \in Q(N, L), f(x) f(T x) \cdots f\left(T^{L-1} x\right)$ is constant, say $\lambda_{I, L} \in G$. Since $h(x)=f(x) h(T x), h(x)=f(x) f(T x) \cdots$ $f\left(T^{L-1} x\right) h\left(T^{L} x\right)$. Hence

$$
h(x)=\lambda_{I, L} \cdot h\left(T^{L} x\right)
$$

holds almost everywhere on $I$. Letting $T^{L} I=J \in \mathcal{P}_{N}$, the map $T^{L}$ : $I \rightarrow J$ is bijective and it is easily shown that

$$
\left|\frac{1}{\mu(I)} \int_{I} h(x) d \mu(x)\right|=\left|\frac{1}{\mu(J)} \int_{J} h(y) d \mu(y)\right| .
$$

Since $Q(N, L)$ measures at least $\frac{1}{2}$, the set of $x$ which is interior to some $I \in Q(N, L)$ for an infinitely number of $L$ must also measures at least $\frac{1}{2}$. Fixing such an $x$, we have that (1) holds. We may assume that $x$ is a Lebesgue point of $h$. Since $\mathcal{P}_{N}$ is finite, it can be assumed $J$ is always the same on the right side of (1). By the Lebesgue density theorem[5], we can assume that the left side of (1) tends to $h(x)$. Hence

$$
|h(x)|=\left|\frac{1}{\mu(J)} \int_{J} h(y) d \mu(y)\right| .
$$

Without loss of generality, we may assume that $|h(x)|=1$. Hence we obtain

$$
\left|\frac{1}{\mu(J)} \int_{J} h(y) d \mu(y)\right|=1 .
$$

Since $|h(y)|=1$ holds almost everywhere, $h(y)$ has to be constant on $J$.

Since $f(x)$ be an $G$-valued step function with finite discontinuity and $T^{N} J=X, h(x)$ is also step function with finite discontinuity.

Proposition 1. Let $E$ be a finite union of intervals. If there exists $c>0$ such that $E \cap[0, c)=[0, c)$, then almost every $x$ is a normal number mod 2 with respect to $E$ on the logistic map.

Proof. Let $\phi(x)=\exp \left(\pi i \mathbf{1}_{E}(x)\right)$ then $\phi(x) \neq 1$ for all $x \in[0, c)$ Suppose that there exists $h(x)$ with $\phi(x) h(S x)=h(x)$. By Lemma 2, we may assume that $h(x)$ is also a step function with finite discontinutiy. Hence there exist $0<r<\min \left(\frac{1}{2}, c\right)$ such that $h(x)$ is constant on $[0, r)$. Thus both $h(x)$ and $h(S x)$ are constant on $\left[0, \frac{1-\sqrt{1-r}}{2}\right)$ and it contradicts the assumption that $\phi(x) \neq 1$ on $[0, c)$.

In the following, we restrict our attention to $\phi(x)=\exp \left(\pi i \mathbf{1}_{E}(x)\right)$ where $E=[a, b]$, i.e., an interval for the similarity of Veech's results[6]. 
In the case of finite union of intervals, we can apply the similar arguments as in the case of an interval.

Theorem 1. For the logistic map, almost every $x$ is a normal number mod 2 with respect to all intervals except for $[a, b]=\left[\frac{1}{4}, 1\right]$ or $[a, b]=$ $\left[\frac{1}{2}-\frac{\sqrt{3}}{4}, \frac{1}{2}+\frac{\sqrt{3}}{4}\right]$.

Proof. Let $\phi(x)=\exp \left(\pi i \mathbf{1}_{E}(x)\right)$. At first, we find a condition that $T_{\phi}$ is not ergodic, i.e., there exist $q(x)$ such that $\phi(x) q(S x)=q(x)$. As in the proof of Lemma 2 , the tent map $T$ on $([0,1], \mu)$ and the logistic map $S$ on $([0,1], \nu)$ are measure theoretically isomorphic via the topological homeomorphism $\psi(x)=\sin ^{2}\left(\frac{\pi x}{2}\right)$ by Lemma 1 . Let $f(x)=\phi(\psi(x))$ and $h(x)=q(\psi x)$. Then $f(x) h(T x)=h(x)$ and $f(x)$ is also a step function with finite discontinuity points. Since $f^{2}(x)=1, f^{2}(x) h^{2}(T x)=h^{2}(x)$ is equivalent to $h^{2}(T x)=h^{2}(x)$. Since $T$ is ergodic, $h^{2}(x)$ is constant. Hence we may assume that $h^{2}(x)=1$. Thus by Lemma $2, h(x)$ has the form of

$$
\left.h(x)=\exp \left(\pi i \sum_{k=1}^{n-1} b_{k} \mathbf{1}_{[} a_{k}, a_{k+1}\right](x)\right)
$$

where $b_{k} \in\{0,1\}$ and $0=a_{1}<a_{2}<\cdots<a_{n}=1$. Note that for any measurable set $F \subset[0,1)$,

$$
T^{-1} F=\frac{1}{2} F \cup\left(1-\frac{1}{2} F\right) .
$$

Since $h(x)$ has $n-2$ discontinuity points and $h(T x)$ has at least $2(n-2)$ discontinuity points, $h(x) \overline{h(T x)}$ has at least $(n-2)$ discontinuity points. Since $f(x)$ has two discontinuity points, we have

$$
0 \leq n-2 \leq 2 .
$$

Hence we have only three cases: $n=2,3,4$. In the case of $n=2, E$ has to be $[0,1), f(x)$ is a constant function and it is an exceptional case. Note that if $g(x)=\lambda h(x)$, then $f(x) g(T x)=g(x)$ also holds. Hence we may also assume that $b_{1}=1$ and $b_{2}=0$.

Let $F=\{x \mid h(x)=-1\}$. Then $f(x) h(T x)=h(x)$ is equivalent to $E=F \triangle T^{-1} F$.

(Case $\mathbf{n}=\mathbf{3}$ ). In this case, $F$ has to be the form of $F=[c, 1]$ with $0<c<1$. Since

$$
[a, b]=F \triangle T^{-1} F=\left(\frac{c}{2}, c\right) \cup\left(1-\frac{c}{2}, 1\right)
$$


modulo measure zero set, we have $c=1-\frac{c}{2}$. Thus $F=\left[\frac{2}{3}, 1\right]$ and $E=\left[\frac{1}{3}, 1\right]$. Therefore for the logistic map,

$$
E=\left[\sin ^{2}\left(\frac{\pi}{6}\right), 1\right]=\left[\frac{1}{4}, 1\right]
$$

with $F=\left[\sin ^{2}\left(\frac{\pi}{3}\right), 1\right]=\left[\frac{3}{4}, 1\right]$.

(Case $\mathbf{n}=4$ ). In this case, $F$ has to be the form of $F=[c, d]$ with $0<c<d<1$. Since

$$
[a, b]=F \triangle T^{-1} F=\left(\frac{c}{2}, c\right) \cup\left(1-\frac{c}{2}, 1\right)=(c, d) \triangle\left[\left(\frac{c}{2}, \frac{d}{2}\right) \cup\left(1-\frac{d}{2}, 1-\frac{c}{2}\right)\right]
$$

modulo measure zero set, we have $\frac{d}{2}=c$ and $1-\frac{d}{2}=d$. Thus $F=\left[\frac{1}{3}, \frac{2}{3}\right]$ and $E=\left[\frac{1}{6}, \frac{5}{6}\right]$. Therefore for the logistic map,

$$
E=\left[\sin ^{2}\left(\frac{\pi}{12}\right), \sin ^{2}\left(\frac{5 \pi}{12}\right)\right]=\left[\frac{1}{2}-\frac{\sqrt{3}}{4}, \frac{1}{2}+\frac{\sqrt{3}}{4}\right]
$$

with $F=\left[\sin ^{2}\left(\frac{\pi}{6}\right), \sin ^{2}\left(\frac{\pi}{3}\right)\right]=\left[\frac{1}{4}, \frac{3}{4}\right]$.

Hence we complete the proof with the following corollary.

Recall that if $\exp \left(\pi i \mathbf{1}_{E}(x)\right) q(T x)=q(x)$ with $q(x)=\exp \left(\pi i \mathbf{1}_{F}(x)\right)$ for some measurable set $F$, then

$$
\lim _{N \rightarrow \infty} \frac{1}{N} \sum_{1}^{N} \exp \left(\pi i d_{n}(x)\right)=q(x) \int_{[0,1)} q(t) d \mu(t) .
$$

Hence we have the following corollary.

Corollary 1. (i) If $E=\left[\frac{1}{4}, 1\right]$, we have

$$
\lim _{N \rightarrow \infty} \frac{1}{N} \sum_{1}^{N} \exp \left(\pi i d_{n}(x)\right)=\frac{1}{3} \exp \left(\pi i \mathbf{1}_{[c, d]}(x)\right)
$$

where $[c, d]=\left[\frac{3}{4}, 1\right]$.

(ii) If $E=\left[\frac{1}{2}-\frac{\sqrt{3}}{4}, \frac{1}{2}+\frac{\sqrt{3}}{4}\right]$, we have

$$
\lim _{N \rightarrow \infty} \frac{1}{N} \sum_{1}^{N} \exp \left(\pi i d_{n}(x)\right)=\frac{1}{3} \exp \left(\pi i \mathbf{1}_{[c, d]}(x)\right)
$$

where $[c, d]=\left[\frac{1}{4}, \frac{3}{4}\right]$. 


\section{References}

[1] Y. Ahn and G. H. Choe, Spectral types of skewed Bernoulli shift, Proc. Amer. Math. Soc. 128 (2000), 503-510.

[2] Y. Ahn, A class of compact group extensions of $\beta$-transformations, J. Math. Anal. Appl. 376 (2011), 154-161.

[3] G. H. Choe, Computational Ergodic Theory, Springer-Verlag, 2005.

[4] G. H. Choe, T. Hamachi and H. Nakada Skew product and mod 2 normal numbers, Studia Math. 165 (2004), 53-60

[5] W. Rudin, Real and Complex Analysis, McGraw-Hill, 1986.

[6] W. A. Veech, strict ergodicity of uniform distribution and Kronecker-Weyl theorem mod 2, Tran. Amer. Math. Soc. 140 (1969), 1-33.

[7] P. Walters, An Introduction to Ergodic Theory, Springer-Verlag, New York, 1982.

\section{Young-Ho Ahn}

Department of Mathematics, Mokpo National University,

534-729, Korea.

E-mail: yhahn@mokpo.ac.kr 